\title{
PERIODICITY OF RECURRING SEQUENCES IN RINGS
}

\author{
TORLEIV KLøVE
}

1.

In this paper all rings are commutative (not necessarily containing a unit). A recurring sequence in a ring $R$ is a sequence $x_{0}, x_{1}, \ldots$ of elements from $R$ satisfying.

$$
x_{n}=P\left(x_{n-1}, \ldots, x_{n-\varrho}\right)+r_{0} \quad \text { for all } n \geqq \varrho,
$$

where $P$ is a polynomial without constant term and with coefficients $r_{1}, r_{2}, \ldots, r_{m}$ in $R$. We call $r_{0} \in R$ "the constant term of the recurring sequence". When $P$ is a polynomial of first degree the sequence is called a linear recurring sequence.

A sequence $x_{0}, x_{1}, \ldots$ in $R$ is called periodic if there exist integers $\mu>0$ and $N \geqq 0$ such that

$$
x_{n+\mu}=x_{n} \quad \text { for all } n \geqq N ;
$$

$\mu$ is then a period for the sequence.

We shall prove the following theorem.

THeORem 1. If the linear recurring sequence defined by $x_{0}=0, x_{n}=x_{n-1}+r_{0}$ (that is $x_{n}=n r_{0}$ ) is periodic and the linear recurring sequences defined by $x_{0}=r, x_{n}=r x_{n-1}$ (that is $x_{n}=r^{n+1}$ ) are periodic for each $r \in R$ then every recurring sequence in $R$ with constant term $r_{0}$ is periodic.

2.

For each $r \in R$ let $S(r)$ be the least positive integer such that $S(r) r=0$. If no such integer exists we put $S(r)=\infty$. We shall need the following lemmas.

LEMMa 1. The following two conditions are equivalent:

(i) For each $r \in R$ the linear recurring sequence defined by $x_{0}=r, x_{n}=r x_{n-1}$ is periodic.

Received May 16, 1972. 
(ii) For each $r \in R$ there exist two positive integers $k(r), l(r)$ such that $r^{k(r)+l(r)}=r^{l(r)}$.

Lemma 2. Let $r_{0} \in R$. The following two conditions are equivalent:

(i) The linear recurring sequence defined by $x_{0}=0, x_{n}=x_{n-1}+r_{0}$ is periodic.

(ii) $S\left(r_{0}\right)$ is finite.

Lemma 3. Let $R$ be a ring satisfying condition (ii) of lemma 1 .

(i) For each $r \in R$ there exists a positive integer $\lambda(r)$ such that $S\left(r^{\lambda(r)}\right)$ is finite.

(ii) If $S(a)$ is finite for some $a \in R$, then $S(a r)$ is finite and divides $S(a)$ for all $r \in R$.

Lemmas 1 and 2 are immediate consequences of the definition of periodicity. To prove lemma 3 we first note that if $r^{k+l}=r^{l}$, then $r^{\alpha k+\lambda}=r^{\lambda}$ for all integers $\alpha \geqq 0$ and $\lambda \geqq l$. Let $\lambda=\lambda(r)=\max (l(r), l(2 r)), k=k(r)$ and $\varkappa=k(2 r)$. Then

$$
2^{\lambda} r^{\lambda}=(2 r)^{\lambda}=(2 r)^{k x+\lambda}=2^{k \varkappa+\lambda} r^{\varkappa k+\lambda}=2^{k \varkappa+\lambda} r^{\lambda} .
$$

Hence

$$
\left(2^{k \varkappa+\lambda}-2^{\lambda}\right) r^{\lambda}=0,
$$

which proves (i). To prove (ii) we note that

$$
S(a) a r=(S(a) a) r=0 .
$$

Hence $S(a r) \leqq S(a)$. Put $S(a)=p S(a r)+q$ where $0 \leqq q<S(a r)$. Then

$$
q a r=S(a) a r-p S(a r) a r=0
$$

and hence $q=0$ by the minimality of $S(a r)$.

We note that if $R$ of lemma 3 is a ring with unit $e$, then $S(r)$ is finite for all $r \in R$. This is a consequence of lemma 3 since $e^{\lambda(e)}=e$, hence $S(e)$ is finite and so $S(r)=S(e r)$ is finite. In particular, the two equivalent conditions of lemma 2 are satisfied for such rings.

3.

We now turn to the proof of theorem 1. Suppose conditions (i) (and hence conditions (ii)) of lemma 1 and 2 are satisfied and let $x_{0}, x_{1}, \ldots$ be any recurring sequence satisfying (1.1). Applying (1.1) repeatedly we get

$$
x_{n}=Q_{n}\left(x_{0}, \ldots, x_{e^{-1}}\right)+r_{0} Q_{n}^{*}\left(x_{0}, \ldots, x_{\varrho^{-1}}\right),
$$


where $Q_{n}$ is a polynomial whose coefficients are polynomials $q_{n j}$, $j=1,2, \ldots, J(n)$, in $r_{1}, r_{2}, \ldots, r_{m}$ with integral coefficients, $r_{1}, r_{2}, \ldots, r_{m}$ being the coefficients of $P$, and $Q_{n}{ }^{*}$ is a polynomial whose coefficients are polynomials $q_{n j}{ }^{*}, j=1,2, \ldots, J^{*}(n)$, in $r_{0}, r_{1}, \ldots, r_{m}$.

The polynomials $Q_{n}$ are given recursively by

$$
\begin{gathered}
Q_{n}\left(x_{0}, \ldots, x_{\varrho_{-1}}\right)=x_{n} \quad \text { if } 0 \leqq n \leqq \varrho-1, \\
Q_{n}\left(x_{0}, \ldots, x_{\varrho_{-1}}\right)=P\left(Q_{n-1}(\ldots), \ldots, Q_{n-\varrho}(\ldots)\right) \quad \text { if } n \geqq \varrho .
\end{gathered}
$$

Let $d(n)$ be the degree of the term in the polynomials $q_{n j}$ of least degree. By (3.2) and (3.3)

$$
\begin{aligned}
& d(n)=0 \quad \text { if } 0 \leqq n \leqq \varrho-1, \\
& d(n) \geqq \min _{1 \leqq i \leqq \varrho}\{d(n-i)+1\} \quad \text { if } n \geqq \varrho .
\end{aligned}
$$

By induction on $n$ we get

$$
d(n) \geqq[n / \varrho]
$$

where $[x]$ denotes the greatest integer $\leqq x$. Put $S=$ least common multiple of $S\left(r_{i}^{\lambda\left(r_{i}\right)}\right), i=1,2, \ldots, m$. Then

$$
S r_{1}^{\alpha_{1}} \ldots r_{m}^{\alpha_{m}}=0
$$

if $\alpha_{i} \geqq \lambda\left(r_{i}\right)$ for at least one $\mathrm{i}$ by lemma 3 . Hence, if

$$
n \geqq \varrho\left\{\lambda\left(r_{1}\right)+\ldots+\lambda\left(r_{m}\right)-m+1\right\}
$$

then, by (3.4), $q_{n j}$ is a polynomial with coefficients $<S$. Since $q_{n j}$ is of degree $<k\left(r_{i}\right)+\lambda\left(r_{i}\right)$ in $r_{i}$, there are only a finite number of such polynomials. Further $Q_{n}$ is a polynomial of degree $<k\left(x_{i}\right)+l\left(x_{i}\right)$ in $x_{i}$, hence there are only a finite number of different $Q_{n}$ 's.

As to the polynomials $r_{0} Q_{n}{ }^{*}$ we note that the coefficients of $r_{0} q_{n j}{ }^{*}$ are $<S\left(r_{0}\right)$, hence there are only a finite number of different $r_{0} Q_{n}{ }^{*}$. Finally, by (3.1), there are only a finite number of different $x_{n}$ 's and so there are only a finite number of different arrays $x_{n}, x_{n+1}, \ldots, x_{n+\ell-1}$. Hence there exist integers $N \geqq 0$ and $\mu>0$ such that

$$
x_{n+\mu}=x_{n} \quad \text { for } n=N, N+1, \ldots, N+\varrho-1 .
$$

By (1.1), $x_{n+\mu}=x_{n}$ for all $n \geqq N$.

4.

Ward [1] defined periodicity modulo an ideal $A$ in $R$ as follows:

The sequence $x_{0}, x_{1}, \ldots$ is periodic modulo $A$ if $x_{n+\mu}-x_{n} \in A$ for all $n \geqq N$. 
This, however, is the same as periodicity of the sequence $x_{0}+A$, $x_{1}+A, \ldots$ in the ring $R / A$. Thus the first part of Ward's theorem 6.1 is a corollary of our theorem 1 .

5.

We may define recurrence somewhat more generally and prove an analogous theorem in the general case.

Let $C$ be a set containing $R$, in which there is defined a multiplication

(i) which extends the multiplication in $R$,

(ii) which is commutative, assosiative, and distributive over addition in $R$,

(iii) such that $c r \in R$ for all $c \in C, r \in R$.

A recurring sequence in $R$ with coefficients in $C$ is a sequence $x_{0}, x_{1}, \ldots$ of elements from $R$ satisfying (1.1) where now $P$ is a polynominal with coefficients in $C$; the $r_{0}$ in (1.1) is still an element of $R$.

A possible choice of $C$ is $C=R \cup Z, Z$ being the set of integers. The multiplication in $C$ is defined in the natural way. This choice of $C$ covers all recurrences with integral coefficients, these would not be otherwise covered if $R$ is a ring without unit.

Another choice is $C$ being a ring having $R$ as an ideal.

We get the following theorem (which reduces to theorem 1 if $C=R$ ).

THEOREM 2. If the linear recurring sequence defined by $x_{0}=0, x_{n}=x_{n-1}+r_{0}$ (that is $x_{n}=n r_{0}$ ) is periodic and the linear recurring sequences defined by $x_{0}=r, x_{n}=c x_{n-1}$ (that is $x_{n}=c^{n} r$ ) are periodic for each $r \in R$ and $c \in C$ then every recurring sequence in $R$ with coefficients in $C$ and constant term $r_{0}($ in $R)$ is periodic.

With minor alterations the proof of theorem 1 also applies to theorem 2.

REFERENCE

1. M. Ward, Arithmetical properties of sequences in ring, Ann. of Math. (2), 39 (1938), 210-219.

UNIVERSITY OF BERGEN, BERGEN, NORWAY 